\title{
Cigarette smoking and depression: tests of causal linkages using a longitudinal birth cohort ${ }^{\dagger}$
}

\author{
Joseph M. Boden, David M. Fergusson and L. John Horwood
}

\section{Background}

Research on the comorbidity between cigarette smoking and major depression has not elucidated the pathways by which smoking is associated with depression.

\section{Aims}

To examine the causal relationships between smoking and depression via fixed-effects regression and structural equation modelling.

\section{Method}

Data were gathered on nicotine-dependence symptoms and depressive symptoms in early adulthood using a birth cohort of over 1000 individuals.

\section{Results}

Adjustment for confounding factors revealed persistent significant $(P<0.05)$ associations between nicotine- dependence symptoms and depressive symptoms. Structural equation modelling suggested that the best-fitting causa model was one in which nicotine dependence led to increased risk of depression. The findings suggest that the comorbidity between smoking and depression arises from two routes; the first involving common or correlated risk factors and the second a direct path in which smoking increases the risk of depression.

\section{Conclusions}

This evidence is consistent with the conclusion that there is a cause and effect relationship between smoking and depression in which cigarette smoking increases the risk of symptoms of depression.

\section{Declaration of interest}

None.
In recent decades, a number of studies have examined the comorbidity of substance use and mental disorders. ${ }^{1-4}$ One particular comorbidity that has been examined in some detail is the association between cigarette smoking and major depression, with a number of epidemiological studies showing that cigarette smoking is frequently comorbid with depression. ${ }^{5-22}$ The apparent comorbidity between cigarette smoking and depression may be explained in at least two ways. First, it could be argued that both smoking and depression are caused by common underlying genetic and environmental factors. Evidence for this position has been provided by studies that have demonstrated shared genetic and environmental influences in the comorbidity of smoking and depression. 5,6,8,10,15,17,19,21 However, a number of these studies have also found evidence for residual associations between cigarette smoking and depression after controlling for shared genetic and environmental influence, suggesting that there may be an association between the two that cannot be accounted for by common confounding factors. ${ }^{6,8,10,15,17,21}$

Second, it could also be argued that cigarette smoking and depression are related in a causal manner, such that either: depression increases the risks of smoking; or smoking increases the risks of depression. Indeed, several studies have argued that cigarette smoking may arise or increase as a result of selfmedication of depressive symptoms. ${ }^{18,21-25}$ On the other hand, further studies have found evidence that smoking increases the risks of depression. ${ }^{12,14-16,21}$

In a previous paper ${ }^{26}$ we have developed a general approach to examining the comorbidity of disorders using a two-stage analytic strategy. In the first stage, fixed-effects regression models were used to control the associations between comorbid conditions, such as smoking and depression, for common, non-observed sources of confounding. These sources of confounding included all non-observed genes and environment, as well as time-dynamic

'See editorial, pp. 425-426, this issue. confounding factors. In the second stage, structural equation modelling methods were used to model reciprocal pathways between the comorbid conditions. The present study employs a similar approach, described below.

Although it is often believed that epidemiological research can only control for the effects of observed confounders, this is not strictly correct and there are a number of analytic approaches that permit the control of non-observed confounders in nonexperimental research. The best known of these is the discordant twin design in which monozygotic twins who are discordant for some exposure variable (e.g. smoking) are compared on an outcome measure (e.g. depression). Since the twin pairs share both common genes and common environment this comparison controls for these factors. ${ }^{19,27}$

The principles underlying the discordant twin design can also be applied to longitudinal data on singletons via the fixed-effects regression model. Subject to the availability of longitudinal data, it proves possible to estimate the associations between a timevarying exposure variable (such as smoking) and a time-varying outcome measure (such as depression) net of any non-observed fixed factors that are associated with the outcome and that may be correlated with the exposure variable. In effect, this model makes it possible to eliminate one major source of confounding from fixed factors. ${ }^{28,29}$ However, the model does not address the issue of confounders that may vary over time and to control for such confounding, the fixed-effects model needs to be augmented by observed time-dynamic confounding factors.

Establishing that smoking and depression are related even following control for confounding is an important step in ascertaining a causal relationship between smoking and depression. However, such analysis does not resolve the issue of the direction of causality: even with well-collected longitudinal data, establishing which factor is antecedent and which factor is consequent proves difficult. ${ }^{18}$ Furthermore, there is a possibility that smoking and depression are reciprocally related to each other 
by a feedback loop in which smoking increases risks of depression, while at the same time the onset of depression leads to an increased consumption of tobacco. ${ }^{21}$ Structural equation models provide one means to address this issue by devising statistical models that permit reciprocal relationships between indices of smoking and depression and using these models to provide a guide to likely patterns of causation. ${ }^{17,30}$

In this paper we address these issues using data gathered over the course of a longitudinal study in which measures of depression and smoking were obtained at regular intervals. In the first stage of the analyses fixed-effects regression modelling was used to control the associations between smoking and depression for common confounding factors including non-observed common genes and environment. In the second stage, methods of structural equation modelling were used to explore the direction of causality.

\section{Method}

\section{Participants}

The data were gathered during the course of the Christchurch Health and Development Study (CHDS). In this study a birth cohort of 1265 children (635 males, 630 females) born in the Christchurch (New Zealand) urban region in mid-1977 has been studied at birth, 4 months, 1 year and annually to age 16 years, and again at ages 18,21 and 25 years. ${ }^{31,32}$ All study information was collected on the basis of signed consent from study participants.

\section{Symptoms of major depression, ages 18, 21 and 25}

At ages 18, 21 and 25 years, study participants were interviewed on a structured mental health interview designed to assess aspects of mental health and psychosocial adjustment. In these assessments, items from the Composite International Diagnostic Interview $(\mathrm{CIDI})^{33}$ pertaining to major depression were used to assess DSM-IV ${ }^{34}$ symptom criteria for major depression, using the full range of questions pertaining to depressive symptoms, over the previous 12 months. For the purposes of the present analyses, the dichotomous responses (yes; no) to each individual symptom item in the symptom reports were summed at ages 18, 21, and 25 to create a measure of the total number of symptoms of depression reported during the year prior to each assessment.

In addition, for the purposes of examining the robustness of the data to alternative methods of measurement using structural equation modelling, participants were classified on three-level ordinal scale measures reflecting the severity of depression symptomatology in each year. This scale was: 0 , had no depressive symptoms; 1 , had depressive symptoms but did not meet criteria for major depression; 2, met criteria for major depression. At age $18,74.5 \%$ of participants reported no symptoms; $7.2 \%$ reported some symptoms; and $18.3 \%$ met criteria for major depression; at age $21,73.6 \%$ of participants reported no symptoms; $8.2 \%$ reported some symptoms; and $18.2 \%$ met criteria for major depression; and at age 25, $79.3 \%$ of participants reported no symptoms; $7.0 \%$ reported some symptoms; and $13.7 \%$ met criteria for major depression.

\section{Cigarette smoking, ages 18, 21 and 25}

At ages 18, 21 and 25, participants were questioned as to the frequency with which they had smoked cigarettes during the month prior to the assessment. Those who reported smoking were further questioned using custom-written survey items to assess DSM-IV symptom criteria for nicotine dependence (see online supplement). For the purposes of the present analyses, the responses to each individual symptom item in the symptom reports were summed for ages 18,21 and 25 to create a measure of the total number of definite symptoms of nicotine dependence reported during the month prior to each of the three assessments. All non-smokers at each age period received scores of no symptoms on the measures of nicotine-dependence symptoms.

In addition, for the purposes of examining the robustness of the data to alternative methods of measurement using structural equation modelling, participants were classified on three-level ordinal scale measures reflecting the severity of nicotinedependence symptomatology in each year. This scale was: 0 , had no nicotine dependence symptoms; 1, had nicotine-dependence symptoms but did not meet criteria for nicotine dependence; 2 , met criteria for nicotine dependence. At age 18, 83.0\% of participants reported no symptoms; $3.1 \%$ reported some symptoms; and $13.9 \%$ met criteria for nicotine dependence; at age $21,63.1 \%$ of participants reported no symptoms; $12.4 \%$ reported some symptoms; and $24.5 \%$ met criteria for nicotine dependence; and at age 25, $65.1 \%$ of participants reported no symptoms; $11.9 \%$ reported some symptoms; and $23.0 \%$ met criteria for nicotine dependence.

In addition, for the purposes of examining whether the findings of the study were robust to alternative methods of measuring nicotine intake, the smoking frequency data described above were used to create a six-point measure reflecting the number of cigarettes participants reported smoking per day. The categories were: non-smoker; $<1$ per day; $1-4$ per day; $5-9$ per day; $10-20$ per day; and 21 plus per day.

\section{Time-dynamic covariate factors}

The following time-dynamic covariate factors were chosen from the database of the study on the basis of: their associations with symptoms of either nicotine dependence or depression in preliminary analyses; and previous research examining smoking and depression among the present cohort. ${ }^{17,35}$ These covariate factors included the following, each assessed during the periods 17-18, 20-21 and 24-25 years.

Stressful life events were assessed by responses to items from the Feeling Bad Scale ${ }^{36}$ and custom-written survey items to provide an index of the number of stressful life events during each year. Anxiety disorder at ages 18, 21 and 25 was measured via items from the (CIDI) ${ }^{33}$ to assess DSM-IV symptom criteria for a range of anxiety disorders. Cannabis use was assessed at each year by questioning the frequency with which participants had used cannabis during each year since the previous assessment. Other illicit drug use was assessed at each year by questioning participants whether they had used illicit drugs other than cannabis during each year since the previous assessment. Affiliation with 'deviant' peers was assessed via questions pertaining to friends' use of alcohol, tobacco or other illicit drugs, or involvement in criminal activity, problems with aggressive behaviour or being in trouble with the law, in the year prior to each assessment. Unemployment was assessed by asking participants about their experience of unemployment in each year and classified into four levels reflecting the duration of unemployment in the year. Partner substance use and criminal offending was assessed on the basis of participant reports of the extent to which their partner: used tobacco, alcohol or illicit drugs or had problems resulting from alcohol or illicit drugs; and engaged in criminal offending, had problems with aggressive behaviour or were in trouble with the law, in the year prior to each assessment.

\section{Statistical methods}

Associations between smoking and depression.

In the first stage of the analysis the association between symptoms of nicotine dependence and depression in each year was assessed 
using a Poisson regression model in which the rates of depressive symptoms were modelled as a log-linear function of nicotine dependence symptoms. In each case the significance of the association was assessed using the Wald chi-squared statistic for the effect of nicotine-dependence symptoms from the fitted model. In addition, the pooled association between nicotinedependence symptoms and depressive symptoms was estimated via generalised estimating equation methods $s^{37,38}$ to fit a population-averaged regression model in which the rate of depressive symptoms at each of the three time periods was modelled as a log-linear function of nicotine-dependence symptoms during each time period. This model was of the form:

$$
\log \left(Y_{i t}\right)=B_{0}+B_{1} X_{i t}
$$

where $\log \left(Y_{i t}\right)$ was the $\log$ rate of depressive symptoms reported by the $i$ th subject in a given interval $t$, and $X_{i t}$ represented nicotinedependence symptoms during the interval $t$. In this model observations from the same individual over time were permitted to be correlated with an unstructured correlation matrix. In addition, the associations were modelled using depressive symptoms as the exposure variable, and nicotine-dependence symptoms as the outcome variable. All models were fitted using Stata 8.0 for Windows. From the fitted models, estimates of the incidence rate ratio (IRR) and 95\% confidence intervals were calculated.

In order to examine the robustness of the data to alternative measures of modelling nicotine intake, further models of the form described above were fitted in which the measure of nicotine dependence was substituted by a measure of daily cigarettesmoking frequency.

\section{Fixed-effects model for covariate adjustment}

To adjust the associations between smoking and depression for unobserved fixed and observed time-dynamic confounding factors, a conditional fixed-effects Poisson regression model was fitted to the joint data over the three measurement periods. This model was of the form:

$$
\log \left(Y_{i t}\right)=\alpha_{i}+B_{1} X_{i t}+\Sigma B_{k} Z_{i k t}
$$

In this model the $\alpha_{i}$ are individual specific terms that are assumed to reflect the effects of all fixed sources of variation in the outcome

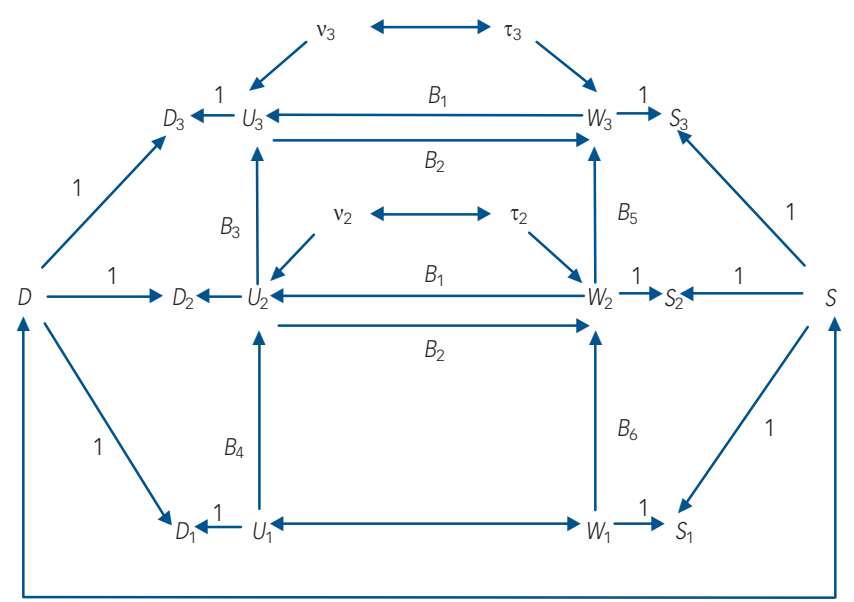

Fig. 1 Autoregressive model of depression and nicotinedependence symptomatology incorporating fixed effects and reciprocal paths between time-dynamic components of depression and nicotine-dependence symptoms.

$D_{t}$, depression symptoms at time $t, S_{t}$, nicotine-dependence symptoms at time $t, D$,

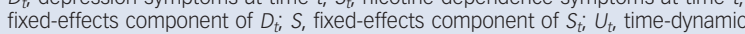
component of $D_{t}, W_{t}$, time-dynamic component of $S t ; v$, disturbance term for $U_{t,}$ disturbance term for $W$
$Y_{i t}$, and $Z_{i k t}$ are the set of observed time-dynamic covariates. The fixed effects $\alpha_{i}$ are assumed to be constant over time and to be correlated with other predictors in the model. The major advantage of the fixed-effects model is that it can adjust for all sources of fixed covariate effects, including non-observed fixed confounders. ${ }^{28}$

In addition to the model described above, the fixed-effects models were also fitted using depressive symptoms as the exposure variable, and nicotine-dependence symptoms as the outcome variable. Also, in order to examine the robustness of the data to alternative measures of modelling nicotine intake, further models of the form described above were fitted in which the measure of nicotine dependence was substituted by a measure of daily cigarette smoking frequency.

\section{Structural equation modelling}

To explore issues of causal direction, a series of structural equation models was fitted to the symptom count measures of depression and nicotine dependence observed for the three intervals 17-18, 20-21 and 24-25 years. These models incorporated both fixed effects influencing the measures of depression and smoking over time and the potential to examine both unidirectional and reciprocal effects between depression and smoking within time intervals.

Figure 1 depicts a model with reciprocal causal effects between depression and smoking. This model assumed that:

(a) the observed symptom measures of depression (denoted $D_{t}$, $t=1,2,3)$ at ages $17-18(t=1), 20-21 \quad(t=2)$ and $24-25$ $(t=3)$ were influenced by fixed sources of variation $(D)$ that were constant over time and by time-dynamic sources of variation $\left(U_{t}\right)$;

(b) the observed symptom measures of nicotine dependence (denoted $S_{t}, t=1,2,3$ ) were also influenced by fixed sources of variation $(S)$ that were constant over time and timedynamic sources of variation $\left(W_{t}\right)$;

(c) the fixed factors $D$ and $S$ were permitted to be correlated;

(d) the time-dynamic components of depressive symptoms $\left(U_{t}\right)$ and nicotine-dependence symptoms $\left(W_{t}\right)$ were linked by autoregressive processes in which past depressive symptoms predicted future depressive symptoms, and past nicotinedependence symptoms predicted future nicotine-dependence symptoms respectively;

(e) the time-dynamic components of depression and nicotinedependence symptoms were reciprocally related at $t=2,3$ so that current $U_{t}$ influenced current $W_{t}$ and vice versa. These reciprocal effects were assumed to be constant over time;

(f) the time-dynamic components $U_{1}$ and $W_{1}$ were assumed to be correlated rather than reciprocally related in order to assist with model identifiability.

In this model the fixed effects $(D, S)$ are latent variables that summarise the net effect of all non-observed fixed factors that exert a constant effect on the measures of depressive symptoms and nicotine-dependence symptoms respectively over time. These factors include all childhood, family and personal characteristics that have a fixed effect on outcomes over time, and thus may include both genetic and environmental influences. The timedynamic components of the model $\left(U_{t}, W_{t}\right)$ represent the effect of all other sources of variance in depressive symptoms and nicotine-dependence symptoms respectively that are not solely due to fixed factors. The equations defining this model were as follows: 
model equations:

$$
\begin{aligned}
& D_{t}=D+U_{t}(t=1,2,3) ; S_{t}=S+W_{t}(t=1,2,3) \\
& U_{3}=B_{1} W_{3}+B_{3} U_{2}+v_{3} ; W_{3}=B_{2} U_{3}+B_{5} W_{2}+\tau_{3} \\
& U_{2}=B_{1} W_{2}+B_{4} U_{1}+v_{2} ; W_{2}=B_{2} U_{2}+B_{6} W_{1}+\tau_{2}
\end{aligned}
$$

model assumptions:

$$
\operatorname{cov}\left(D, U_{t}\right)=\operatorname{cov}\left(D, W_{t}\right)=\operatorname{cov}\left(S, U_{t}\right)=\operatorname{cov}\left(S, W_{t}\right)=0(t=1,2,3)
$$

$\operatorname{cov}\left(D, v_{t}\right)=\operatorname{cov}\left(D, \tau_{t}\right)=\operatorname{cov}\left(S, v_{t}\right)=\operatorname{cov}\left(S, \tau_{t}\right)=0 \quad(t=2,3)$

$\operatorname{cov}\left(v_{t}, \tau_{s}\right)=\operatorname{cov}\left(v_{t}, v_{s}\right)=\operatorname{cov}\left(\tau_{t}, \tau_{s}\right)=0$ for $t \neq s(t, s=2,3)$

$\operatorname{cov}\left(D_{t}, v_{s}\right)=\operatorname{cov}\left(D_{t}, \tau_{s}\right)=\operatorname{cov}\left(S_{t}, v_{s}\right)=\operatorname{cov}\left(S_{t}, \tau_{s}\right)=0$ for $t<s$

$\operatorname{cov}\left(U_{t}, v_{s}\right)=\operatorname{cov}\left(U_{t}, \tau_{s}\right)=\operatorname{cov}\left(W_{t}, v_{s}\right)=\operatorname{cov}\left(W_{t}, \tau_{s}\right)=0$ for $t<s$

In these equations the terms $v_{t}$ and $\tau_{t}(t=2,3)$ represent disturbance terms reflecting unexplained sources of variation in the time-dynamic components of depressive symptoms $\left(U_{t}\right)$ and nicotine-dependence symptoms $\left(W_{t}\right)$ respectively. The coefficients $B_{1}, B_{2}$ represent the reciprocal effects of nicotine-dependence symptoms on depressive symptoms and vice versa respectively within each time period. The coefficients $B_{3}, B_{4}$ and $B_{5}, B_{6}$ represent the across-time stabilities in the time-dynamic components of depressive symptoms and nicotine-dependence symptoms respectively.

The reciprocal cause model depicted in Fig. 1 was fitted to the observed measures of depressive symptoms and nicotinedependence symptoms at ages 17-18, 20-21, 24-25 years. The fit of this model was then compared with the fit of two other models that assumed unidirectional causal effects between depression and nicotine-dependence symptoms. These models were: a model that assumed a unidirectional effect from nicotine-dependence symptoms to depressive symptoms (i.e. $B_{1} \neq 0 ; B_{2}=0$ ); a model that assumed a unidirectional effect from depressive symptoms to nicotine-dependence symptoms (i.e. $\left.B_{1}=0 ; B_{2} \neq 0\right)$.

Since the observed measures were markedly non-normally distributed the models were fitted to the covariance matrix of the observed data using the method of weighted least squares. All models were fitted using LISREL 8 for Windows. (Scientific Software International; www.ssicentral.com). Model goodness of fit was assessed on the basis of a number of indices including the following three: the model chi-squared goodness of fit statistic; the root mean squared error of approximation (RMSEA). Values of RMSEA less than 0.05 are assumed to be indicative of a well-fitting model; and the comparative fit index (CFI). This index varies between 0 and 1 with values close to 1 indicating a well-fitting model.

To examine the sensitivity of the results to model estimation methods a series of alternative models was fitted to the data. These included: a model that used the ordered categorical measures of nicotine dependence and depression described above; and a model that substituted a measure of cigarette-smoking frequency in place of the measure of nicotine dependence.

\section{Sample size and sample bias}

The present analyses are based on the sample of 1055 participants for whom data on cigarette smoking and depression symptomatology were available on at least one occasion from 18, 21 and 25 years. However, since not all participants were assessed at all ages the observed sample numbers varied between age 18 $(n=1025)$, age $21(n=1011)$ and age $25(n=1003)$. These samples represented between 79 and $81 \%$ of the initial cohort of 1265 participants.

To adjust for possible sample selection bias resulting from sample attrition, a two-stage process was used. In the first instance, a sample selection model was constructed by using data gathered at birth to predict inclusion in the analysis sample. This showed that there were statistically significant $(P<0.05)$ tendencies for the obtained sample to underrepresent children from more socially disadvantaged backgrounds (low parental education, low socioeconomic status, single-parent family). On the basis of the fitted selection models, the sample was then post-stratified into a series of groups and the probability of study participation estimated for each group.

For the Poisson regression models the data were then re-analysed with the observations for each individual weighted by the inverse of the probability of study participation, and using standard error estimates that were robust to the weighting procedures used. For the structural equation models analyses were conducted that both assumed that missing observations within the analysis sample were missing at random and weighted the observed data by the inverse of the probability of study participation. The analyses were conducted using weighted least squares procedures that were robust to the data-weighting and missingdata assumptions. In all cases the analyses produced essentially identical conclusions to the findings reported here, suggesting that the effects of missing data and selection bias on the results were likely to be minimal.

\section{Results}

\section{Associations between nicotine-dependence symptoms and symptoms of depression, ages 17-25}

Table 1 shows the associations between the number of symptoms of nicotine dependence and rates of depressive symptoms at ages $17-18,20-21$, and $24-25$ years (the distribution of the threecategory scales for nicotine dependence and smoking in Table 1 are shown in online Table DS1). In addition, Table 1 also reports on the pooled mean number of depressive symptoms over the three measurement periods, and provides an estimate of the IRRs and 95\% confidence intervals, derived from Poisson regression models (see Methods) for the pooled (populationaveraged) association between the symptoms of nicotine dependence and symptoms of depression at ages 17-18, 20-21, and 24-25. The Table shows that, at each age, increasing levels of nicotinedependence symptoms were significantly $(P<0.0001)$ associated with increasing rates of depressive symptoms, with these trends were reflected in the measures of nicotine dependence and depressive symptoms pooled over the three observation periods (likelihood ratio LR $\chi^{2}(1)=387.25, P<0.0001$ ).

Overall, those reporting at least five symptoms of nicotine dependence had rates of depressive symptoms that were 2.13 times (95\% CI 1.98-2.31) those of individuals who reported no symptoms of nicotine dependence.

In addition, Poisson regression models in which symptoms of nicotine dependence were regressed on depressive symptoms produced comparable results (LR $\chi^{2}(1)=108.21, P<0.0001$ ). Also, analyses in which a measure of daily cigarette intake was used in place of the measure of nicotine-dependence symptoms produced comparable results (depressive symptoms regressed on cigarette intake: LR $\chi^{2}(1)=223.86, P<0.0001$; cigarette intake regressed on depressive symptoms: LR $\left.\chi^{2}(1)=25.47, P<0.0001\right)$.

\section{Adjustment for covariate factors}

The associations between nicotine-dependence symptoms and depressive symptoms were adjusted for non-observed genetic and environmental factors using fixed-effects regression methods. Fixed-effects models were fitted to the data for nicotine-dependence symptoms and depressive symptoms at ages 
$17-18,20-21$, and $24-25$, and extended to include a series of observed time-dynamic covariate factors measured during the period 17-25 years (see Methods). Adjustment for fixed and time-dynamic covariate factors reduced the magnitude of the pooled association between nicotine-dependence symptoms and depressive symptoms ( $\operatorname{LR} \chi^{2}(1)=22.54, P<0.0001$ ), but the association remained statistically significant.

In addition, fixed-effects models with time-dynamic covariate factors, in which symptoms of nicotine dependence were regressed on depressive symptoms, again showed a statistically significant association between nicotine-dependence symptoms and depressive symptoms ( $\left.\operatorname{LR} \chi^{2}(1)=4.34, P<0.05\right)$. Again, analyses in which a measure of daily cigarette intake was used in place of the measure of nicotine-dependence symptoms produced comparable results (depressive symptoms regressed on cigarette intake: LR $\chi^{2}(1)=21.89, P<0.0001$; cigarette intake regressed on depressive symptoms: $\left.\operatorname{LR} \chi^{2}(1)=868, P<0.05\right)$.

\section{Results from structural equation models}

The findings above are consistent with the view that nicotinedependence symptoms and depressive symptoms may be linked by a cause and effect model. However, the analysis does not establish that this association is one in which increasing frequency of nicotine-dependence symptoms leads to increased frequency of depressive symptoms or vice versa. To address this issue, a series of structural equation models was fitted to the data to test alternative assumptions about the direction of association between nicotinedependence symptoms and depressive symptoms (see Methods). Specifically, three models were fitted:

(a) Model 1: a model assuming a reciprocal association between depressive symptoms and nicotine-dependence symptoms within time;

(b) Model 2: a model assuming a unidirectional casual effect from nicotine-dependence symptoms to depressive symptoms; (c) Model 3: a model assuming a unidirectional causal effect from depressive symptoms to nicotine-dependence symptoms.

In fitting these models the correlations shown in Fig. 1 between the disturbance terms $v_{t}$ and $\tau_{t}$ were fixed to zero as preliminary analyses showed that these coefficients were non-significant for all models and at all times.

Table 2 shows estimates of the effects of nicotine-dependence symptoms and depressive symptoms on each other and associated goodness of fit statistics from the three models. The analyses suggested the following conclusions. First, Models 1 and 2 were well fitting and led to the same general conclusion: nicotinedependence symptoms were significantly related to depressive symptoms $(P<0.005)$ but depressive symptoms were not significantly related to nicotine-dependence symptoms $(P=0.21)$. The difference in chi-squared statistics for the two models was not statistically significant $\left(\Delta \chi^{2}(1)=0.15, P=0.70\right)$ suggesting that the path from depressive symptoms to nicotinedependence symptoms could be constrained to zero without affecting model fit. Consistent with these results Model 3 was significantly less well fitting than Models 1 and 2. In particular, the change in model chi-squared from Models 1 to 3 was significant $\left(\Delta \chi^{2}(1)=4.19, P=0.04\right)$, suggesting that the pathway from nicotine-dependence symptoms to depressive symptoms could not be constrained to zero without affecting model fit.

As noted in Methods these estimates were obtained from models utilising continuous-count measures of DSM-IV symptom criteria for nicotine dependence or depression in each year (online Table DS1). To examine the sensitivity of the results to model estimation methods a series of alternative models was fitted to the data. These models included: a model that used ordered categorical measures of nicotine dependence and depression; and a model that substituted a measure of daily cigarette intake for the measure of nicotine dependence symptoms. Each of these models produced results consistent with the conclusions drawn above.

\begin{tabular}{|c|c|c|c|c|c|}
\hline \multirow[b]{2}{*}{ Depressive symptoms measure } & \multicolumn{5}{|c|}{ Number of nicotine dependence symptoms } \\
\hline & 0 & $1-2$ & $3-4$ & $5-6$ & $P^{\mathrm{b}}$ \\
\hline \multicolumn{6}{|l|}{ Depressive symptoms, mean (s.d.) } \\
\hline Age $17-18$ & $1.57(3.00)$ & $1.88(3.30)$ & $2.96(3.78)$ & $5.38(4.09)$ & $<0.0001$ \\
\hline Age 20-21 & $1.44(2.88)$ & $1.85(3.29)$ & $2.57(3.44)$ & $2.94(3.87)$ & $<0.0001$ \\
\hline Age $24-24$ & $1.32(2.83)$ & $0.89(2.52)$ & $1.55(3.21)$ & $2.46(3.64)$ & $<0.0001$ \\
\hline Depressive symptoms, pooled mean (s.d.) & $1.45(2.91)$ & $1.51(3.06)$ & $2.31(3.49)$ & $3.15(3.95)$ & $<0.0001$ \\
\hline Pooled association, incidence rate ratio $(95 \% \mathrm{Cl})$ & 1 & $1.29(1.26-1.32)$ & $1.66(1.58-1.75)$ & $2.13(1.98-2.31)$ & \\
\hline
\end{tabular}

\begin{tabular}{|c|c|c|c|c|c|}
\hline \multirow[b]{2}{*}{ Model } & \multicolumn{2}{|c|}{ Model parameter } & \multicolumn{3}{|c|}{ Goodness of fit indices } \\
\hline & $B$ (s.e.) & $P$ & Chi squared & RMSEA & CFI \\
\hline \multicolumn{6}{|l|}{ Model 1: reciprocal effects } \\
\hline Effect of nicotine-dependence symptoms on depressive symptoms $\left(B_{1}\right)$ & $0.246(0.095)$ & 0.004 & $\chi^{2}(5)=6.24, P=0.28$ & 0.014 & 0.999 \\
\hline Effect of depressive symptoms on nicotine dependence symptoms $\left(B_{2}\right)$ & $-0.025(0.031)$ & 0.21 & & & \\
\hline \multicolumn{6}{|l|}{ Model 2: unidirectional } \\
\hline Effect of nicotine-dependence symptoms on depressive symptoms $\left(B_{1}\right)$ & $0.181(0.052)$ & $<0.001$ & $\chi^{2}(6)=6.39, P=0.38$ & 0.010 & 1.00 \\
\hline \multicolumn{6}{|l|}{ Model 3: unidirectional } \\
\hline Effect of depressive symptoms on nicotine-dependence symptoms $\left(B_{2}\right)$ & $0.047(0.016)$ & $<0.01$ & $\chi^{2}(6)=10.43, P=0.10$ & 0.032 & 0.996 \\
\hline
\end{tabular}




\section{Discussion}

In this analysis we have used data gathered over the course of a longitudinal study to examine the comorbidities of major depression and nicotine dependence. The analysis used advanced statistical modelling methods to control for non-observed sources of confounding and to explore causal pathways. The analysis led to the following conclusions.

First, in agreement with previous research, ${ }^{5-22}$ there was evidence of significant comorbidity between nicotine-dependence symptoms and depressive symptoms at ages 18, 21 and 25. Second, adjustment for non-observed fixed sources of confounding and observed time-dynamic covariate factors showed that the association between depression and smoking could not be entirely explained by these sources of confounding. In addition, further analyses in which nicotine-dependence symptoms were regressed on depressive symptoms produced similar estimates. Also, analyses in which the measure of nicotine dependence was replaced by a measure of daily cigarette intake produced comparable results, suggesting that the findings were robust to alternative methods of measurement. In general, these findings are consistent with previous research that has concluded that the comorbidity between cigarette smoking and depression cannot be explained by common sources of confounding, including common genes and common environment. ${ }^{6,8,10,15,17,21}$ These findings are also consistent with the view that there is likely to be a direct cause and effect association between smoking and depression.

To explore possible pathways between cigarette smoking and depression, methods of structural equation modelling were used to fit a reciprocal causation model. This analysis suggested that the best-fitting model was one in which there was a unidirectional association from symptoms of nicotine dependence to depressive symptoms, but no reverse effect from depressive symptoms to nicotine dependence. Again, analyses using either an alternative measure of nicotine dependence and depression, or a measure of daily cigarette intake in place of nicotine dependence, produced similar results, suggesting that the models were robust to alternative methods of measurement. Collectively this evidence is consistent with the conclusion that there is a cause and effect relationship between smoking and depression in which cigarette smoking increases the risk of symptoms of depression. ${ }^{12,14-16,21}$ The underlying mechanisms that give rise to such an association are unclear; however, it has been proposed that this linkage may arise from the effects of nicotine on neurotransmitter activity in the brain, causing changes to neurotransmitter activity and leading to increased risk of depression. ${ }^{39,40}$

It should also be noted that the findings of the present study are not in agreement with a number of studies that have suggested a causal (self-medication) pathway from depression to smoking. ${ }^{18,21-23,25}$ There are several reasons why the findings may differ from those of other studies. First, some studies have not tested assumptions regarding causal pathways using structural models. ${ }^{18,21}$ Second, other studies have used retrospective recall, ${ }^{22}$ whereas the present study was prospective in nature. Third, at least one study modelled the associations between smoking and internalising disorders, rather than depression per se. ${ }^{23}$ Finally, a further study found evidence for a causal link from depression to smoking only among a subset of participants with a particular genetic variant. $^{25}$

The findings of the present study are also not in agreement with a number of studies that have failed to find a persistent association between cigarette smoking and major depression., ${ }^{5,19,41}$ Again, there may be a number of reasons why the findings of the present study differ from previous findings. For example, one study employed a design in which a subsample of participants was selected for further study at subsequent time points, ${ }^{5}$ whereas another study compared a selected sample of currently depressed smokers with currently depressed non-smokers. ${ }^{41}$ In addition, one study employed retrospective recall for measures of depression and smoking. ${ }^{19}$

\section{Limitations}

Finally, it is important to recognise that the conclusions drawn in this analysis rely on some underlying assumptions that are necessary to identify the models we have presented. The most pervasive of these assumptions is that the pattern of comorbidity being studied is represented by a stable causal process that was operative throughout the course of this study. This is clearly a strong assumption, but it is essential for both the fixed-effects and reciprocal-causes models. Further research may be required to examine whether the assumptions regarding the stability of patterns of comorbidity between smoking and depression are correct. Furthermore, it is likely that the models that we have used to represent these data are only approximations to a more complex set of conditions. For these reasons the findings of this study should be viewed as suggestive rather than definitive.

Joseph M. Boden, PhD, David M. Fergusson, PhD, L. John Horwood, MSC, Department of Psychological Medicine, Christchurch Health and Development Study, University of Otago, Christchurch School of Medicine and Health Sciences, Christchurch, New Zealand

Correspondence: David M. Fergusson, Christchurch Health and Development Study, University of Otago, Christchurch School of Medicine and Health Sciences, PO Box 4345, Christchurch, New Zealand. Email: dm.fergusson@otago.ac.nz

First received 9 Mar 2009, final revision 25 May 2009, accepted 16 Sep 2009

\section{Funding}

This research was funded by grants from the Health Research Council of New Zealand, the National Child Health Research Foundation, the Canterbury Medical Research Foundation and the New Zealand Lottery Grants Board.

\section{References}

1 Smart D, Hayes A, Sanson A, Toumbourou JW. Mental health and wellbeing of Australian adolescents: pathways to vulnerability and resilience. Int J Adolesc Med Health 2007; 19: 263-8.

2 Jane-Llopis E, Matytsina I. Mental health and alcohol, drugs and tobacco: a review of the comorbidity between mental disorders and the use of alcohol, tobacco and illicit drugs. Drug Alcohol Rev 2006; 25: 515-36.

3 Rehm J, Taylor B, Room R. Global burden of disease from alcohol, illicit drugs and tobacco. Drug Alcohol Rev 2006; 25: 503-13.

4 Siegfried N. A review of comorbidity: major mental illness and problematic substance use. Aust N Z J Psychiatry 1998; 32: 707-17.

5 Rohde $\mathrm{P}$, Kahler CW, Lewinsohn PM, Brown RA. Psychiatric disorders, familial factors, and cigarette smoking: II. Associations with progression to daily smoking. Nicotine Tob Res 2004; 6: 119-32.

6 Haarasilta LM, Marttunen MJ, Kaprio JA, Aro HM. Correlates of depression in a representative nationwide sample of adolescents (15-19 years) and young adults (20-24 years). Eur J Public Health 2004; 14: 280-5.

7 Benjet C, Wagner FA, Borges GG, Medina-Mora ME. The relationship of tobacco smoking with depressive symptomatology in the Third Mexican National Addictions Survey. Psychol Med 2004; 34: 881-8.

8 Hu MC, Davies M, Kandel DB. Epidemiology and correlates of daily smoking and nicotine dependence among young adults in the United States. Am J Public Health 2006; 96: 299-308.

9 Araya R, Gaete J, Rojas G, Fritsch R, Lewis G. Smoking and common mental disorders: a population-based survey in Santiago, Chile. Soc Psychiatry Psychiatr Epidemiol 2007; 42: 874-80. 
10 Mykletun A, Overland S, Aaro LE, Liabo HM, Stewart R. Smoking in relation to anxiety and depression. Evidence from a large population survey: the HUNT study. Eur Psychiatry 2008; 23: 77-84.

11 Wiesbeck GA, Kuhl HC, Yaldizli O, Wurst FM. Tobacco smoking and depression - results from the WHO/ISBRA study. Neuropsychobiology 2008; 57: 26-31.

12 Steuber TL, Danner F. Adolescent smoking and depression: which comes first? Addict Behav 2006; 31: 133-6.

13 Compton WM, Thomas YF, Stinson FS, Grant BF. Prevalence, correlates, disability, and comorbidity of DSM-IV drug abuse and dependence in the United States: results from the national epidemiologic survey on alcohol and related conditions. Arch Gen Psychiatry 2007; 64: 566-76.

14 Klungsoyr O, Nygard JF, Sorensen T, Sandanger I. Cigarette smoking and incidence of first depressive episode: an 11-year, population-based follow-up study. Am J Epidemiol 2006; 163: 421-32.

15 Munafo MR, Hitsman B, Rende R, Metcalfe C, Niaura R. Effects of progression to cigarette smoking on depressed mood in adolescents: evidence from the National Longitudinal Study of Adolescent Health. Addiction 2008; 103 162-71

16 Pasco JA, Williams L, Jacka FN, Ng F, Henry MJ, Nicholson GC, et al. Tobacco smoking as a risk factor for major depressive disorder: population-based study. Br J Psychiatry 2008; 193: 322-6.

17 Fergusson DM, Goodwin RD, Horwood L. Major depression and cigarette smoking: results of a 21 year longitudinal study. Psychol Med 2003; 33: 1357-67.

18 Breslau N, Kilbey M, Andreski P. Nicotine dependence and major depression: new evidence from a prospective investigation. Arch Gen Psychiatry 1993; 50: 31-5.

19 Kendler KS, Neale MC, MacLean CJ, Heath AC, Eaves LJ, Kessler RC. Smoking and major depression. A causal analysis. Arch Gen Psychiatry 1993; 50: 36-43.

20 Breslau N. Psychiatric comorbidity of smoking and nicotine dependence. Behav Genet 1995; 25: 95-101.

21 Breslau N, Peterson EL, Schultz LR, Chilcoat HD, Andreski P. Major depression and stages of smoking. A longitudinal investigation. Arch Gen Psychiatry 1998; 55: 161-6.

22 Patton GC, Hibbert M, Rosier MJ, Carlin JB, Caust J, Bowes G. Is smoking associated with depression and anxiety in teenagers? Am J Public Health 1996; 86: 225-30.

23 Crone MR, Reijneveld SA. The association of behavioural and emotional problems with tobacco use in adolescence. Addict Behav 2007; 32: 1692-8.

24 Pomerleau CS, Pomerleau OF. Euphoriant effects of nicotine in smokers. Psychopharmacology (Berl) 1992; 108: 460-5.
25 Lerman C, Caporaso N, Main D, Audrain J, Boyd NR, Bowman ED, et al. Depression and self-medication with nicotine: the modifying influence of the dopamine D4 receptor gene. Health Psychol 1998; 17: 56-62.

26 Fergusson DM, Boden JM, Horwood LJ. Tests of causal linkages between alcohol abuse/dependence and major depression. Arch Gen Psychiatry 2009; 66: $260-6$.

27 Kendler KS. Of genes and twins. Psychol Med 2003; 33: 763-8.

28 Hamerle A, Ronning G. Panel analysis for qualitative variables. In Handbook of Statistical Modeling for the Social and Behavioral Sciences (eds G Arminger, CC Clogg, ME Sobel): 401-51. Plenum Press, 1995.

29 Hausman J, Hall BH, Griliches Z. Econometric models for count data with an application to the patents $-\mathrm{R} \& \mathrm{D}$ relationship. Econometrica 1984; $\mathbf{5 2}$ 909-38.

30 Kendler KS, Gardner CO, Prescott CA. Toward a comprehensive developmental model for major depression in women. Focus 2005; 3: 83-97.

31 Fergusson DM, Horwood $\amalg$. The Christchurch Health and Development Study: review of findings on child and adolescent mental health. Aust NZ Psychiatry 2001; 35: 287-96.

32 Fergusson DM, Horwood $\sqcup$, Shannon FT, Lawton JM. The Christchurch Child Development Study. A review of epidemiological findings. Paediatr Perinatal Epidemiol 1989; 3: 278-301.

33 World Health Organization. Composite International Diagnostic Interview (CIDI). World Health Organization, 1993.

34 American Psychiatric Association. Diagnostic and Statistical Manual of Mental Disorders (4th edn) (DSM-IV). APA, 1994.

35 Fergusson DM, Lynskey MT, Horwood LJ. The role of peer affiliations, social, family and individual factors in continuities in cigarette smoking between childhood and adolescence. Addiction 1995; 90: 647-60.

36 Lewis CE, Seigel JM, Lewis MA. Feeling bad: exploring sources of distress among pre-adolescent children. Am J Public Health 1984; 74: 117-22.

37 Liang K-Y, Zeger SL. Longitudinal data analysis using generalized linear models. Biometrika 1986; 73: 13-22.

38 Zeger SL, Liang K-Y. Longitudinal data analysis for discrete and continuous outcomes. Biometrics 1986; 42: 121-30.

39 Haustein KO, Haffner S, Woodcock BG. A review of the pharmacological and psychopharmacological aspects of smoking and smoking cessation in psychiatric patients. Int J Clin Pharmacol Ther 2002; 40: 404-18.

40 Picciotto MR, Caldarone BJ, King SL, Zachariou V. Nicotinic receptors in the brain. Links between molecular biology and behavior. Neuropsychopharmacology 2000; 22: 451-65.

41 Roy K, Parker G, Mitchell P, Wilhelm K. Depression and smoking: examining correlates in a subset of depressed patients. Aust N Z J Psychiatry 2001; 35 329-35 